\title{
Diabetes gestacional: um algoritmo de tratamento multidisciplinar
}

\author{
Gestational diabetes management: a \\ multidisciplinary treatment algorithm
}

Letícia Schwerz Weinert', Sandra Pinho Silveiro ${ }^{1,2}$, Maria Lúcia

Oppermann ${ }^{3}$, Cristiano Caetano Salazar ${ }^{3}$, Bárbara Marina

Simionato ${ }^{4}$, Aline Siebeneichler ${ }^{4}$, Angela Jacob Reichelt ${ }^{2}$

\section{SUMÁRIO}

O tratamento do diabetes gestacional é importante para evitar a morbimortalidade materno-fetal. O objetivo deste artigo é descrever o tratamento atualmente disponível para o manejo otimizado da hiperglicemia na gestação e sugerir um algoritmo de tratamento multidisciplinar. A terapia nutricional é a primeira opção de tratamento para as gestantes, e a prática de exercício físico leve a moderado deve ser estimulada na ausência de contraindicações obstétricas. O tratamento medicamentoso está recomendado quando os alvos glicêmicos não são atingidos ou na presença de crescimento fetal excessivo à ultrassonografia. 0 tratamento tradicional do diabetes gestacional é a insulinoterapia, embora mais recentemente a metformina venha sendo considerada uma opção segura e eficaz. A monitorização do tratamento é realizada com aferição da glicemia capilar e com avaliação da circunferência abdominal fetal por meio de ultrassonografia obstétrica a partir da 28 ${ }^{a}$ semana de gestação. Arq Bras Endocrinol Metab. 2011;55(7):435-45

\author{
Programa de Pós-graduação em \\ Ciências Médicas, Endocrinologia, \\ Universidade Federal do \\ Rio Grande do Sul (UFRGS), \\ Porto Alegre, RS, Brasil \\ 2 Serviço de Endocrinologia, \\ Hospital de Clínicas de Porto \\ Alegre, Porto Alegre, RS, Brasi \\ ${ }^{3}$ Serviço de Ginecologia \\ e Obstetrícia, Hospital de \\ Clínicas de Porto Alegre, \\ Porto Alegre, RS, Brasil \\ ${ }^{4}$ Faculdade de Medicina, UFRGS, \\ Porto Alegre, RS, Brasil
}

\section{Descritores}

Diabetes gestacional; diabetes melito; gestação de alto risco; tratamento

\section{SUMMARY}

Effective treatment of gestational diabetes is important as an attempt to avoid unfavorable maternal and fetal outcomes. The objective of this paper is to describe the available therapies to optimize gestational diabetes treatment and to suggest a multidisciplinary approach algorithm. Nutrition therapy is the first option for the majority of these pregnancies; light to moderate physical activity is recommended in the absence of obstetrical contraindications. Medical treatment is recommended if glycemic control is not achieved or if excessive fetal growth is detected by ultrasound. Insulin is the standard treatment although oral antidiabetic drugs have recently been considered an effective and safe option. The monitoring of gestational diabetes treatment includes capillary glucose measurements and evaluation of fetal abdominal circumference by ultrasound performed around the $2^{\text {th }}$ gestational week. Arq Bras Endocrinol Metab. 2011;55(7):435-45

\author{
Correspondência para: \\ Letícia Schwerz Weinert \\ Serviço de Endocrinologia, \\ Hospital de Clínicas de Porto Alegre \\ Rua Ramiro Barcelos, 2350, \\ prédio $12,4^{\circ}$ andar \\ 90035-903 - Porto Alegre, RS, Brasil \\ leticiasweinert@yahoo.com.br \\ Recebido em 19/Dezl/2010 \\ Aceito em 29/Set/2011
}

\section{Keywords}

Gestational diabetes; diabetes mellitus; high risk pregnancy; treatment

\section{INTRODUÇÃO}

$\mathrm{O}$ diabetes gestacional é definido como qualquer grau de redução da tolerância à glicose, cujo início ou detecção ocorre durante a gravidez $(1,2)$. Sua prevalência é variável, dependendo dos critérios diagnósticos empregados e da população estudada (1). No Brasil, em torno de 7\% das gestações são complicadas pela hiperglicemia gestacional (3).
Habitualmente, o diagnóstico do diabetes gestacional é realizado por busca ativa, com testes provocativos que empregam sobrecarga de glicose, a partir do segundo trimestre da gestação. Mais recentemente, tem-se recomendado a triagem precoce de gestantes de alto risco na primeira consulta pré-natal, o que permite identificar casos de diabetes preexistente e que não devem, portanto, ser rotulados como diabetes gestacional $(1,4)$. 
Embora a maior parte dos casos de diabetes gestacional se resolva no período pós-parto, a definição se aplica independentemente de a condição persistir após a gravidez (1).

$\mathrm{O}$ risco de desfechos adversos maternos, fetais e neonatais aumenta de forma contínua com a elevação da glicemia materna (5). As complicações mais frequentemente associadas ao diabetes gestacional são - para a mãe: a cesariana e a pré-eclâmpsia; - para o concepto: a prematuridade, a macrossomia, a distocia de ombro, a hipoglicemia e a morte perinatal $(5,6)$. Em 2008, o estudo HAPO (Hyperglycemia and Adverse Pregnancy Outcomes) demonstrou não existir um ponto de corte único acima do qual o risco de desfechos adversos estaria mais elevado; eventos desfavoráveis ocorrem, inclusive, com glicemias consideradas normais (5). A partir dos resultados daquele estudo, a rediscussão dos critérios diagnósticos tem sido proposta, visando uniformizar a sobrecarga de glicose e os valores glicêmicos para o diagnóstico do diabetes gestacional.

A International Association of Diabetes and Pregnancy Study Groups (IADPSG), que reúne pesquisadores de todo o mundo, sugeriu novo critério diagnóstico, baseado no teste oral de tolerância com $75 \mathrm{~g}$ de glicose (TTG $75 \mathrm{~g}$ ) realizado em 2 horas (pelo menos um ponto alterado: jejum: $92 \mathrm{mg} / \mathrm{dL}$; lh: $180 \mathrm{mg} /$ $\mathrm{dL} ; 2 \mathrm{~h}: 153 \mathrm{mg} / \mathrm{dL})$. Essa proposta resultaria em aumento significativo da prevalência de casos de diabetes gestacional, chegando a $17 \%$ das gestações (4). A American Diabetes Association (ADA) endossou esse critério diagnóstico em 2011 (1), embora a Sociedade Brasileira de Diabetes (SBD) (7) ainda recomende o emprego do TTG 75 g com coleta de três pontos, havendo necessidade de dois pontos elevados para fazer o diagnóstico de diabetes gestacional (jejum: $95 \mathrm{mg} / \mathrm{dL}$; lh: $180 \mathrm{mg} / \mathrm{dL} ; 2 \mathrm{~h}: 155 \mathrm{mg} / \mathrm{dL})$. No Hospital de Clínicas de Porto Alegre (HCPA), o procedimento diagnóstico adotado até o momento é o preconizado pela $2^{\mathrm{a}}$ Reunião do Grupo de Trabalho de Diabetes e Gravidez, que recomenda o uso do TTG $75 \mathrm{~g}$ em 2 horas, considerando como limites: glicemia de jejum $\geq 110 \mathrm{mg} /$ $\mathrm{dL}$ e/ou glicemia de 2 horas maior $\geq 140 \mathrm{mg} / \mathrm{dL}$ (8). O TTG $75 \mathrm{~g}$ com três pontos também tem sido realizado no HCPA para possibilitar posterior comparação entre critérios e apoiar posicionamento logo que as entidades brasileiras endossem o novo critério da IADPSG (4).

O diagnóstico precoce e o tratamento do diabetes gestacional visam reduzir a morbimortalidade materna e fetal. O estudo de Crowther e cols. demonstrou que o tratamento do diabetes gestacional reduz a morbidade perinatal e melhora a qualidade de vida materna pós-natal (6). Em outro estudo, o tratamento da alteração glicêmica em casos de diabetes gestacional considerados leves - glicemia de jejum abaixo de $95 \mathrm{mg} / \mathrm{dL}$ - reduziu o risco de macrossomia fetal, distocia de ombro, cesariana, e hipertensão durante a gestação, embora não tenha reduzido a mortalidade perinatal (9).

À semelhança do que é feito para os outros tipos de diabetes, o tratamento do diabetes gestacional inclui dieta individualizada e prática de atividade física e, quando indicado, o uso de medicação. A prescrição de dieta e de atividade física constitui-se na etapa inicial $\mathrm{e}$, se as metas glicêmicas preconizadas não forem alcançadas em duas semanas, o tratamento farmacológico é instituído (7). O tratamento padrão para o diabetes gestacional é a insulinoterapia subcutânea. Entretanto, o uso de insulina é complexo, de maior custo e de menor aceitação pelas pacientes; pode, adicionalmente, acarretar ganho indesejado de peso. Como alternativa terapêutica, medicações orais mostraram-se, recentemente, de efetividade comparável à da insulina no tratamento do diabetes gestacional $(10,11)$.

Este artigo tem como objetivo descrever as diferentes modalidades de tratamento que podem ser empregadas no diabetes gestacional e a rotina para acompanhamento dessas gestantes durante o pré-natal. Ao final, um algoritmo de manejo multidisciplinar é sugerido.

\section{TRATAMENTO DO DIABETES GESTACIONAL}

\section{Dieta}

A terapia nutricional é a primeira opção de tratamento para a maioria das gestantes com diabetes gestacional $(12,13)$. Essa terapia evita o ganho excessivo de peso pelas gestantes, além de gerar menor taxa de macrossomia fetal e de complicações perinatais $(12,14)$.

O cálculo de calorias da dieta e do ganho de peso durante a gestação é baseado no peso ideal pré-gestacional das mulheres (Tabela 1) $(12,13,15)$. A dieta prescrita deve conter $30 \mathrm{kcal}$ por $\mathrm{kg}$ de peso ideal, com adição de $340 / 450 \mathrm{kcal}$ no terceiro trimestre.

A ingestão de carboidratos deve ser restrita a menos de $42 \%$ das calorias diárias, com o restante distribuído entre proteínas e gorduras $(12,16)$, na medida em que a evidência demonstra que as gestantes que realizam essa restrição apresentam melhor controle glicêmico pós-prandial, menor necessidade de adição de insulina, 
menor incidência de macrossomia fetal e de cesariana $(16,17)$. Além disso, os carboidratos complexos e com baixo índice glicêmico - massas, pães integrais e cereais com alto teor de fibras - devem ser priorizados $(12,17)$.

O valor calórico total da dieta é distribuído em três refeições e dois a três lanches (12). Em gestantes obesas, os lanches podem ser eliminados. O café da manhã deve conter em torno de $10 \%$ das calorias do dia; o almoço, 30\%; o jantar, 30\%; e os lanches, 30\% (18).

Dietas muito restritivas (com menos de $1.500 \mathrm{kcal} /$ dia) podem induzir cetonemia e não são recomendadas. Dietas com restrição calórica moderada (1.600 - 1.800 kcal) não acarretam cetose, são efetivas no controle do ganho de peso materno e no controle glicêmico $(12,19)$. A redução de calorias da dieta em $50 \%$ tem benefício semelhante àquela com redução em $33 \%$ para o controle glicêmico; a última não acarreta cetose e, portanto, é a restrição recomendada $(15,19)$. A ingestão mínima de carboidratos recomendada na gestação é de $175 \mathrm{~g}$ por dia (15).

Tabela 1. Recomendações para o ganho semanal de peso na gestação de acordo com 0 índice de massa corporal (IMC) pré-gestacional $\left(2^{\circ}\right.$ e $3^{\circ}$ trimestres)

\begin{tabular}{lcc}
\hline IMC pré-gestação $\mathbf{( k g / \mathbf { m } ^ { 2 } )}$ & $\begin{array}{c}\text { Ganho peso total } \\
\mathbf{( k g )}\end{array}$ & $\begin{array}{c}\text { Taxa ganho peso/ } \\
\mathbf{s e m}(\mathbf{k g})\end{array}$ \\
\hline Baixo peso $(<18,5)$ & $12,5-18$ & $0,51(0,44-0,58)$ \\
Peso normal $(18,5-24,9)$ & $11,5-16$ & $0,42(0,35-0,50)$ \\
Sobrepeso $(25-29,9)$ & $7-11,5$ & $0,28(0,23-0,33)$ \\
Obesidade $(\geq 30)$ & $5-9$ & $0,22(0,17-0,27)$ \\
\hline
\end{tabular}

A recomendação sugere ganho de peso de 0,5 a $2 \mathrm{~kg} \mathrm{no} 1^{\circ}$ trimestre. Adaptado de 20 .

\section{Exercício}

A prática de exercícios na gestação tem como benefício a redução da glicemia (12), a redução do ganho excessivo de peso materno e a diminuição da incidência de macrossomia fetal (21). Dessa forma, deve ser recomendada para todas as gestantes diabéticas, na ausência de contraindicações (Tabela 2).

As pacientes que realizavam exercícios previamente à gestação podem continuar ativas (21). Nos casos de diabetes gestacional, recomenda-se realizar 15 a 30 minutos de atividade diária, em cicloergômetro, ou caminhadas em $50 \%$ da capacidade aeróbica da paciente $(12,13,22)$. Deve ser realizada a monitorização da atividade fetal e, idealmente, da glicemia capilar antes e após a atividade. Exercícios não devem ser realizados se a movimentação fetal for menor que dez vezes em
$24 \mathrm{~h}$ e se a glicemia capilar estiver abaixo de $60 \mathrm{mg} / \mathrm{dL}$ ou acima de $250 \mathrm{mg} / \mathrm{dL}(2 \mathrm{l})$.

Tabela 2. Contraindicações à prática de exercício físico durante a gestação

\begin{tabular}{ll}
\hline Absolutas & \multicolumn{1}{c}{ Relativas } \\
\hline Ruptura membranas & Aborto espontâneo prévio \\
Trabalho parto pré-termo & Parto pré-termo prévio \\
Doença hipertensiva gestação & Doença cardiovascular leve-moderada \\
Incompetência istmocérvica & Anemia $(\mathrm{Hb}<10 \mathrm{~g} / \mathrm{dL})$ \\
Crescimento fetal restrito & Doença respiratória leve-moderada \\
Gestação múltipla ( $\geq$ trigemelar) & Desnutrição ou distúrbio alimentar \\
Placenta prévia > 28 $8^{\text {a }}$ semana & Gestação gemelar $>28^{a}$ semana \\
Sangramento persistente $2^{0}$ ou $3^{0}$ trim. & Neuropatia diabética autonômica \\
Diabetes tipo 1, doença tireoidiana, & grave \\
cardiovascular, respiratória ou & Outras condições médicas relevantes \\
sistêmica descompensadas & \\
Retinopatia proliferativa & \\
Neuropatia diabética autonômica & \\
grave & \\
Hipoglicemias graves assintomáticas & \\
Algumas modalidades de exercícios* &
\end{tabular}

* Mergulho com descompressão, exigência de equilíbrio, risco de queda ou trauma abdominal. Adaptado de 23.

\section{Tratamento medicamentoso}

$\mathrm{Na}$ figura 1, está apresentado o algoritmo para o tratamento medicamentoso. Quando um ou mais valores de glicemia capilar estiverem além dos alvos terapêuticos (jejum < $95 \mathrm{mg} / \mathrm{dL}, 1 \mathrm{~h}<140 \mathrm{mg} / \mathrm{dL}$ e 2 $\mathrm{h}<120 \mathrm{mg} / \mathrm{dL}$ ) (l) após duas semanas com medidas de mudança de estilo de vida, ou quando a avaliação ecográfica mostrar sinais de crescimento fetal excessivo, indica-se o tratamento farmacológico $(22,24,25)$.

Uma glicemia de jejum $>140 \mathrm{mg} / \mathrm{dL}$ e/ou uma glicemia de $2 \mathrm{~h}>200 \mathrm{mg} / \mathrm{dL}$ no diagnóstico do diabetes gestacional indica alteração mais grave da tolerância à glicose e o tratamento com medidas não farmacológicas isolado é aplicado por uma semana, com reavaliação mais precoce dos alvos glicêmicos.

As diretrizes, tanto nacionais quanto internacionais, divergem sobre o tratamento medicamentoso do diabetes gestacional. A SBD sugere o uso de insulina como tratamento padrão (7), enquanto o National Institute for Health and Clinical Excellence (NICE) recomenda o uso de metformina (22) e a International Diabetes Federation (IDF) aponta a metformina e a glibenclamida como opções de tratamento, principalmente em situações em que o uso de insulina é difícil (2).

Na rotina de tratamento do HCPA (Figura 1), indica-se a metformina como o medicamento preferencial, na ausência de contraindicações; a insulina é a segunda opção terapêutica e tem sido empregada em casos de 
falha de tratamento, contraindicações à metformina ou de preferência pela paciente.
Na tabela 3, estão apresentadas as principais características de cada medicação que pode ser usada na gestação.

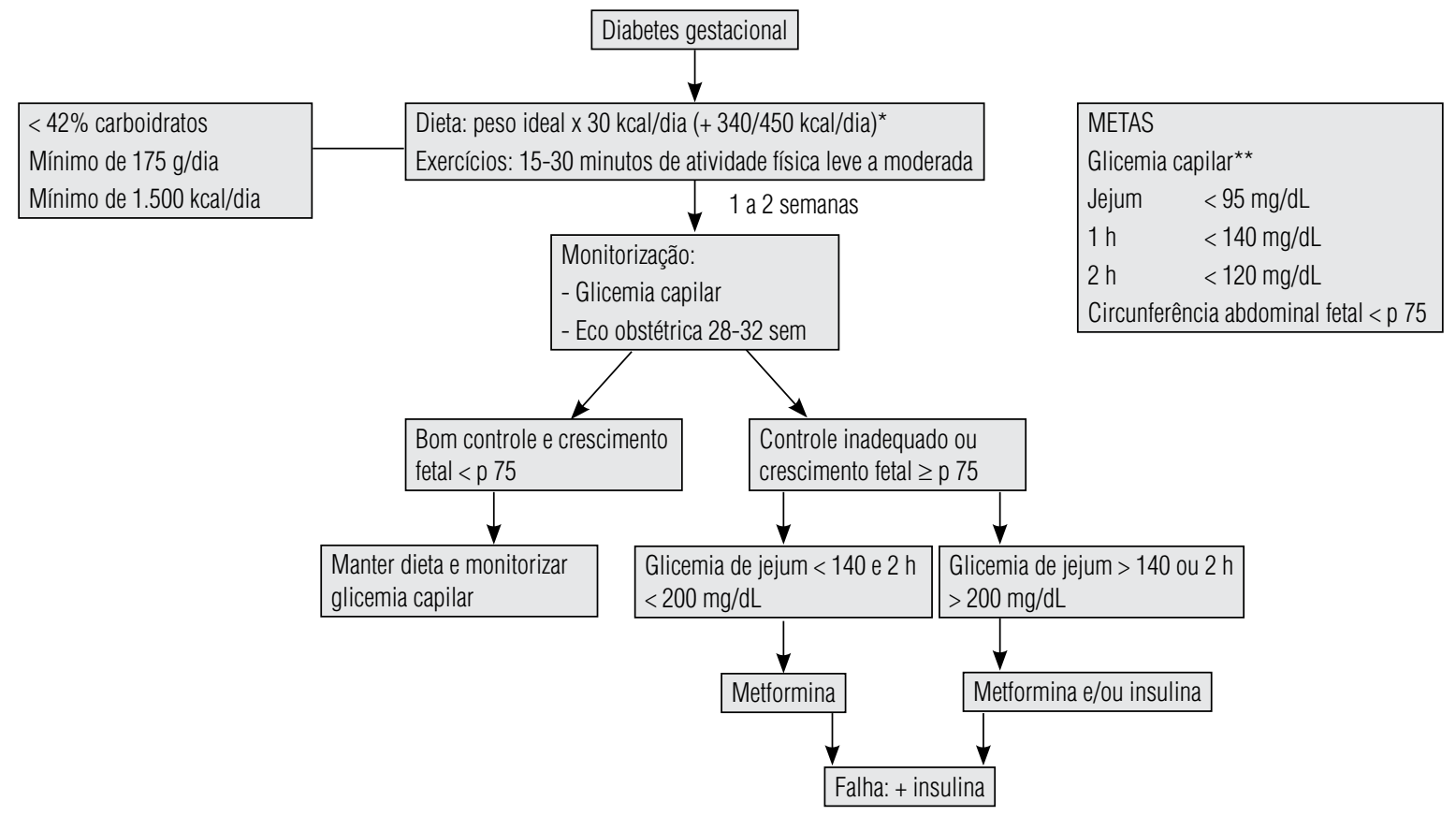

Figura 1. Algoritmo de tratamento do diabetes gestacional. ${ }^{*}$ Terceiro trimestre; ${ }^{* *}$ Adaptado de $1 \mathrm{e} 7$.

Tabela 3. Medicações de uso na gestação

\begin{tabular}{|c|c|c|c|c|c|c|c|}
\hline $\begin{array}{l}\text { Medicação/ } \\
\text { Categoria FDA }\end{array}$ & $\begin{array}{l}\text { Mecanismo de } \\
\text { ação }\end{array}$ & $\begin{array}{l}\text { Passagem } \\
\text { placentária }\end{array}$ & $\begin{array}{c}\text { Transporte } \\
\text { ativo } \\
\text { feto-materno }\end{array}$ & $\begin{array}{l}\text { Eficácia/ } \\
\text { Segurança na } \\
\text { gestação }\end{array}$ & Contraindicações & $\begin{array}{c}\text { Efeitos } \\
\text { adversos }\end{array}$ & $\begin{array}{l}\text { Dose inicial/ } \\
\text { dose máxima }\end{array}$ \\
\hline Metformina/B & $\begin{array}{l}\downarrow \text { Produção } \\
\text { hepática de } \\
\text { glicose }\end{array}$ & Sim & P-gp & $\begin{array}{l}\text { Semelhante à } \\
\text { insulina }\end{array}$ & $\begin{array}{c}\text { TFG }<30 \text { mL/min, } \\
\text { ICC, insuficiência } \\
\text { hepática, DPOC }\end{array}$ & $\begin{array}{l}\text { Náusea, diarreia, } \\
\text { acidose lática } \\
\text { (raro) }\end{array}$ & $\begin{array}{c}500 \mathrm{mg} 1 \mathrm{x} / \mathrm{dia} / \\
2.500 \mathrm{mg} \mathrm{em} \\
3 \mathrm{x} / \mathrm{dia}\end{array}$ \\
\hline $\begin{array}{l}\text { Glibenclamida/ } \\
\text { B/C }\end{array}$ & $\begin{array}{l}\text { Secretagogo de } \\
\text { insulina }\end{array}$ & Insignificante & $\begin{array}{c}\text { BRCP, MRP } 1,2 \text { e } \\
3\end{array}$ & $\begin{array}{l}\text { Semelhante à } \\
\text { insulina }\end{array}$ & $\begin{array}{c}\text { TFG }<50 \text { mL/min, } \\
\text { insuficiência } \\
\text { hepática }\end{array}$ & Hipoglicemia & $\begin{array}{l}2,5 \mathrm{mg} \text { 1x/dia/ } \\
20 \mathrm{mg} \text { em } 2 \text { a } 3 \\
\text { x/dia }\end{array}$ \\
\hline Acarbose/B & $\begin{array}{l}\text { Retardo na } \\
\text { absorção dos } \\
\text { carboidratos }\end{array}$ & Insignificante & Não descrito & $\begin{array}{l}\text { Risco teórico de } \\
\text { prematuridade }\end{array}$ & $\begin{array}{c}\text { Doença intestinal, } \\
\text { cirrose, } \\
\text { insuficiência } \\
\text { hepática }\end{array}$ & $\begin{array}{c}\text { Flatulência, dor } \\
\text { abdominal e } \\
\text { diarreia }\end{array}$ & $\begin{array}{c}50 \mathrm{mg} \text { 1x/dia/ } \\
300 \mathrm{mg} \mathrm{em} \mathrm{3x/dia}\end{array}$ \\
\hline $\begin{array}{l}\text { Insulina } \\
\text { NPH, Regular, } \\
\text { Lispro e Asparte: } \\
\text { B } \\
\text { Glulisina, } \\
\text { Glargina e } \\
\text { Detemir: C }\end{array}$ & $\begin{array}{c}\text { Suplementação } \\
\text { exógena de } \\
\text { insulina }\end{array}$ & $\begin{array}{c}\text { Ausente, exceto } \\
\text { em complexo } \\
\text { antígeno- } \\
\text { anticorpo }\end{array}$ & Não descrito & & - & $\begin{array}{l}\text { Hipoglicemia e } \\
\text { ganho de peso }\end{array}$ & \\
\hline
\end{tabular}

FDA: Food and Drug Administration; TFG: taxa de filtração glomerular; ICC: insuficiência cardíaca congestiva; DPOC: distúrbio pulmonar obstrutivo-crônico; BRCP: cancer resistance protein; MRP1, 2 , 3: human multidrug resistance proteins; P-gp: P glycoprotein. Categorias de risco FDA: A: estudos controlados em gestantes não evidenciam risco fetal. B: não há evidência de risco em humanos. Estudos em animais mostraram risco, mas em humanos não; ou se não há estudos adequados em humanos, os achados em animais são negativos. C: não se pode afastar risco, não há estudos em humanos e animais ou os achados em estudos em animais mostram risco fetal. Avaliar risco/benefício. D: evidência de risco fetal em humanos. Benefícios potenciais podem superar os riscos. X: contraindicado na gestação. Estudos em humanos ou animais ou observações pós-venda mostram risco fetal que claramente supera qualquer possível benefício à gestante. Adaptado de 26 e 27. 


\section{Metformina}

A metformina tem sido considerada uma medicação segura para uso durante toda a gestação (22), inclusive no primeiro trimestre, a partir de estudos realizados primordialmente em mulheres portadoras da síndrome de ovários policísticos (28).

No diabetes gestacional, o uso da metformina apresenta eficácia e segurança semelhantes às da insulina nos desfechos da gestação. No controle glicêmico, a suplementação com insulina pode ser necessária em praticamente metade das gestantes e é mais comumente necessária em mulheres obesas e com hiperglicemia de jejum (11). A droga é bem tolerada na gestação e é pouco frequente a necessidade de redução da dose $(8,8 \%$ das gestantes) ou a suspensão da medicação $(1,9 \%)$ por efeitos adversos (11).

As gestantes candidatas ao uso dessa medicação são aquelas na faixa etária de 18 a 45 anos, com idade gestacional entre 20 e 33 semanas e glicemia de jejum < $140 \mathrm{mg} / \mathrm{dL}$ (11).

\section{Glibenclamida}

A glibenclamida é considerada a melhor opção entre as sulfonilureias para utilização em gestantes, sendo considerada segura para emprego a partir do segundo trimestre de gestação $(10,29,30)$.

No diabetes gestacional, o uso de glibenclamida atingiu eficácia semelhante à da insulina para o controle glicêmico materno e para os desfechos da gestação (10). O único estudo desenhado para comparar a eficácia de glibenclamida em relação à metformina demonstrou controle glicêmico semelhante, porém com menor falha de tratamento (16,2\% com glibenclamida e 34,7\% com metformina) e menor taxa de cesariana não eletiva ( 2 casos com glibenclamida e 11 casos com metformina) com a glibenclamida (31). As candidatas ao uso de glibenclamida são aquelas com gestação única, glicemia de jejum $<140 \mathrm{mg} / \mathrm{dL}$ e idade gestacional entre $11 \mathrm{e}$ 33 semanas (10).

A falha em atingir os níveis glicêmicos adequados é maior nas mulheres com hiperglicemia de jejum (> $110 \mathrm{mg} / \mathrm{dL}$ ) ou com valores acima de $200 \mathrm{mg} / \mathrm{dL}$ em 2 horas no teste de rastreamento (32). Se os alvos glicêmicos não são atingidos em duas semanas com a dose máxima de glibenclamida, recomenda-se trocar o tratamento para insulina (10). Outra opção a ser considerada nesta última situação é a adição de metformina ao tratamento, embora esta conduta não tenha sido avaliada em ensaios clínicos.

\section{Acarbose}

A acarbose reduz a absorção intestinal de amidos e menos de $2 \%$ da dose administrada é absorvida. Estudos animais mostraram ausência de teratogenia em doses 32 vezes maiores que a dose terapêutica humana (26). Até o momento, somente um ensaio clínico randomizado com número reduzido de gestantes mostrou efetividade e desfechos semelhantes aos da insulina (30). Uma potencial restrição ao uso da acarbose poderia ser o risco de aumento de parto pré-termo em decorrência da redução da absorção de carboidratos intestinais, que aumentaria a lise bacteriana e as concentrações de butirato, com consequente aumento na secreção de prostaglandina $\mathrm{E}$ e aumento prematuro da atividade uterina (26). Mais estudos são necessários para avaliar a influência da acarbose sobre os desfechos materno-fetais.

\section{Insulina}

A insulinoterapia é o tratamento padrão do diabetes gestacional devido às comprovadas eficácia e segurança. O tratamento com insulina é iniciado nas gestantes que não atingem as metas de controle glicêmico com dieta ou apresentam falha do tratamento com medicação oral ou apresentam fatores preditores de falha com esses, como glicemia de jejum $>140 \mathrm{mg} / \mathrm{dL}$ ou glicemia de $2 \mathrm{~h}>200 \mathrm{mg} / \mathrm{dL}$ no TTG $75 \mathrm{~g}$.

A dose e o tipo de insulina utilizada dependem do padrão da hiperglicemia: o predomínio da hiperglicemia de jejum ou pré-prandial indica insulina de longa ação, como a NPH; o predomínio da hiperglicemia pós-prandial indica insulina de ação rápida. A dose sugerida é de 1,5 U/10 g carboidratos no café da manhã e de $1 \mathrm{U} / \mathrm{l} 0 \mathrm{~g}$ carboidrato no almoço e no jantar ou, alternativamente, de 0,6 a $1,0 \mathrm{U} / \mathrm{kg} /$ dia, com aumentos progressivos até se alcançar o controle preconizado em relação à glicemia capilar. Algumas gestantes podem necessitar de doses de até $2 \mathrm{U} / \mathrm{kg} /$ dia, especialmente quando a gravidez está próxima do termo ou se são mulheres obesas. A sugestão é administrar 50\%-60\% da dose diária como insulina NPH e 40\%-50\% como insulina rápida (33). Nas hiperglicemias pré e pós-prandiais, sugere-se esquema de tratamento intensivo com quatro doses diárias de insulina, já que houve melhor controle glicêmico e menor morbidade neonatal do que nas gestantes com esquema de apenas duas doses diárias (33).

As insulinas humanas (NPH e regular) são as preferidas por serem menos imunogênicas, além de terem eficácia e segurança comprovadas. Entre as insulinas rá- 
pidas, a lispro e a asparte foram semelhantes à insulina regular, tanto no controle glicêmico quanto na formação de anticorpos (13). Meta-análise recente mostrou que os análogos de rápida e longa ação não foram superiores às insulinas convencionais no controle glicêmico e na taxa de hipoglicemia em gestantes com diabetes melito (34).

As insulinas glargina, detemir e glulisina não foram avaliadas por ensaios clínicos randomizados em gestantes (13). O estudo ex-vivo de placentas a termo, retiradas de gestações sem intercorrências, demonstrou ausência de transferência placentária da insulina glargina; entretanto, é necessária cautela ao extrapolar esses dados para gestações de primeiro trimestre e para gestantes diabéticas (35).

\section{ACOMPANHAMENTO PRÉ-NATAL}

\section{Monitorização metabólica}

Após o início de tratamento com dieta e exercício, e principalmente após o início da terapia medicamentosa, deve ser realizada a monitorização da glicemia capilar para confirmar a adequação do tratamento. As metas do controle glicêmico estão apresentadas na figura 1 .

A avaliação da glicemia de jejum isoladamente não é capaz de detectar os casos de controle inadequado causados por hiperglicemias pós-prandiais. O controle pós-prandial somado ao da glicemia de jejum mostrou-se mais eficaz em reduzir as taxas de cesariana, de macrossomia e de hipoglicemia neonatal (36). Por isso, é necessário aferir a glicemia capilar ao acordar, antes do almoço e do jantar, além daquelas pós-prandiais em 1 ou em 2 horas (13,36). Essa monitorização deve ser idealmente diária (37). Quando não é possível, as gestantes são encaminhadas para o perfil glicêmico semanal em regime de hospital-dia.

Em reanálise recente do estudo Metformin In Gestational Diabetes (MiG), as glicemias em jejum e pós-prandial relacionaram-se expressivamente a complicações perinatais e maternas, independentemente do tipo de tratamento utilizado. Os valores que resultaram em menor número de complicações materno-fetais foram valores inferiores a $88 \mathrm{mg} / \mathrm{dL}$ para a glicemia de jejum e inferiores a $115 \mathrm{mg} / \mathrm{dL}$ para a glicemia $2 \mathrm{~h}$ pós-prandial (38). Por outro lado, estudo de Langer e cols. mostrou que um controle mais estrito, com glicemia média abaixo de $86 \mathrm{mg} / \mathrm{dL}$, determinou aumento na ocorrência de recém-nascidos de baixo peso (39).
A monitorização com o teste $\mathrm{Alc}$ não foi validada com desfechos clínicos no diabetes gestacional, apesar de já estar descrita uma forte associação entre a hemoglobina glicada e glicemias capilares pré e pós-prandiais em gestantes diabéticas (40). A medida basal do teste Alc, ao diagnóstico do diabetes gestacional, está associada com o aumento da taxa de recém-nascidos grande para idade gestacional $(38,40)$ e de pré-eclâmpsia $(38)$. As variações fisiológicas da hemoglobina na gravidez (41) parecem comprometer o desempenho do teste Alc como ferramenta principal de monitoração metabólica.

A medida da cetonúria não está recomendada na avaliação de rotina das mulheres com diabetes gestacional, porém pode ser utilizada para monitorização daquelas que estão sob restrição de carboidratos. Em geral, a cetonúria que ocorre devido ao jejum ou à restrição de carboidratos é leve e não indica, necessariamente, modificação no tratamento da gestante. Entretanto, nas gestantes com glicemia $>240 \mathrm{mg} / \mathrm{dL}$, sintomas de diabetes descompensado, intercorrência com doença grave durante a gestação ou perda de peso, está recomendada a avaliação da cetonúria (13). Estudo observacional sugeriu que o nível intelectual dos filhos das gestantes diabéticas possa estar inversamente relacionado aos níveis maternos de um marcador de cetonúria grave, o $\beta$-hidroxibutirato, no terceiro trimestre (42).

\section{Monitorização do crescimento fetal}

A resposta fetal ao controle da glicose materna pode ser estimada pela medida da circunferência abdominal fetal em ultrassonografias seriadas a partir de 28 semanas de gestação $(13,22)$ e repetidas a cada duas a quatro semanas $(13,22,24,37)$. Considera-se indicador de crescimento fetal excessivo - e marcador indireto do hiperinsulinismo fetal - a medida da circunferência abdominal fetal igual ou superior ao percentil 75 para a idade gestacional (13). Há evidência recente de que duas ultrassonografias consecutivas, realizadas entre 28 e 34 semanas de gestação, são suficientes para excluir a presença de crescimento fetal excessivo, desde que as medidas da circunferência abdominal fetal estejam abaixo do percentil 90 e não existam outros fatores de risco maternos para a macrossomia fetal (obesidade, macrossomia fetal prévia, hiperglicemia em jejum no TTG) (43).

A flexibilização dos alvos glicêmicos maternos conforme a resposta fetal mostrou-se eficaz em reduzir desfechos adversos perinatais $(13,24,25)$ : diante de uma medida de circunferência abdominal fetal igual 
ou superior ao percentil 75 para a idade gestacional, os alvos glicêmicos passam para $80 \mathrm{mg} / \mathrm{dL}$ em jejum e $100-110 \mathrm{mg} / \mathrm{dL}$ em $2 \mathrm{~h}$ pós-prandial, enquanto em fetos com crescimento adequado à idade gestacional os alvos glicêmicos podem ser menos rígidos. Tal flexibilização mostrou redução da incidência de fetos grandes para idade gestacional, sem aumento de fetos pequenos para idade gestacional $(24,25)$.

\section{Rotina pré-natal}

O algoritmo do acompanhamento obstétrico das gestantes portadoras de diabetes gestacional é mostrado na figura 2 .

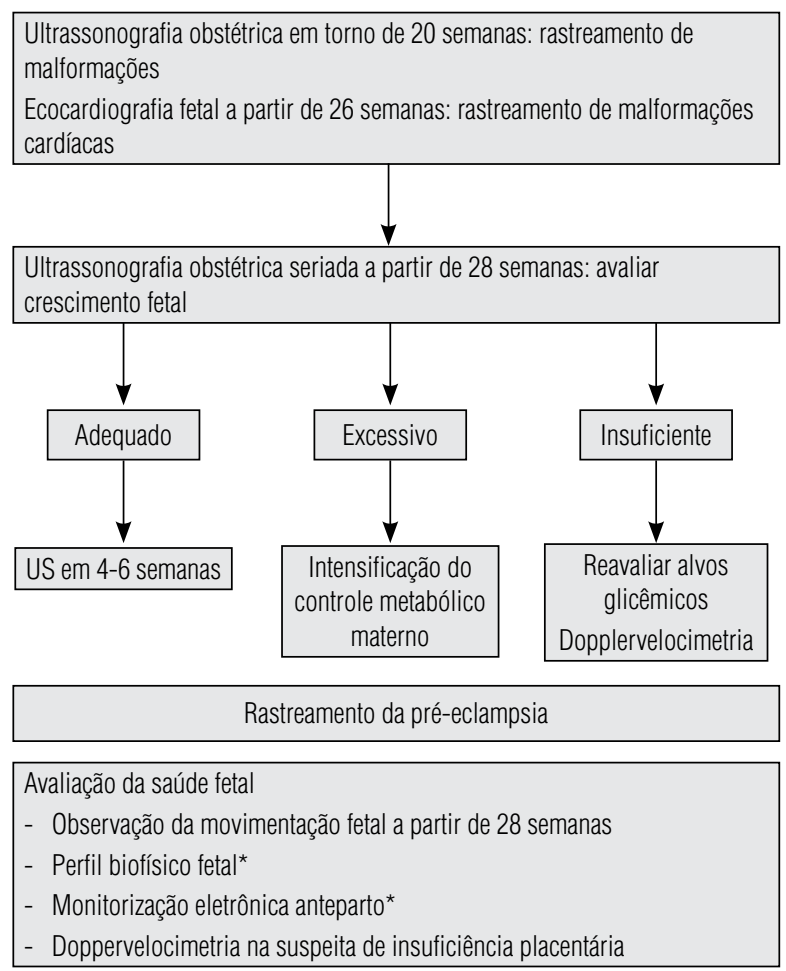

* A partir de 28 semanas em gestante com comorbidade; 32 semanas em gestante em tratamento farmacológico; 37-38 semanas em gestante em tratamento nutricional exclusivo.

Figura 2. Algoritmo de acompanhamento obstétrico em mulheres com diabetes gestacional.

As mulheres em tratamento não farmacológico e com bom controle glicêmico, na ausência de outras indicações obstétricas, podem ser avaliadas a cada duas semanas até a $36^{\text {a }}$ semana de gestação, quando o controle obstétrico passa a ser semanal. As mulheres em uso de insulina ou de antidiabéticos orais são avaliadas a cada semana, eventualmente a intervalos de duas semanas até a $32^{\mathrm{a}}$ semana e, então, semanalmente até o parto.
As ultrassonografias de rotina indicadas durante a gestação - de primeiro trimestre para datação e rastreamento de cromossomopatias (11-14 semanas) e de segundo trimestre para avaliação morfológica fetal (1822 semanas) - devem ser realizadas, idealmente, em todas as gestações.

O ecocardiograma fetal, solicitado para as gestantes com diabetes a partir das 25-26 semanas, visa ao diagnóstico específico de eventuais malformações cardíacas, mais frequentes na gestação com diabetes (44). Entretanto, a tendência atual é a de reservar o exame ecocardiográfico com especialista aos casos suspeitos de anomalia na visão das quatro câmaras cardíacas e vias de saída do ventrículo esquerdo durante o exame de avaliação morfológica fetal (22). A controvérsia sobre o custo-efetividade da ecocardiografia fetal em relação à ecografia morfológica alargada nas gestações de risco para malformações cardíacas tem um ponto crucial: o treinamento do ecografista, já que a detecção de anormalidades com a visão das quatro câmaras cardíacas durante ultrassonografia morfológica foi de $55 \%$ para os ecografistas treinados e de $5,3 \%$ para os não treinados (45).

Para a avaliação da vitalidade fetal, as gestantes são orientadas a observar a movimentação fetal a partir da $28^{a}$ semana de gestação e a buscar atendimento obstétrico se a movimentação fetal estiver reduzida ou ausente. A partir da $32^{\text {a }}$ semana, sugere-se avaliação da vitalidade fetal de uma a duas vezes por semana, guiada primordialmente pelo nível de controle glicêmico obtido, pois não há evidência disponível sobre a efetividade dos testes de bem-estar fetal na gestação com diabetes. A monitorização eletrônica fetal anteparto (MAP) e o perfil biofísico fetal (PBF) são os instrumentos mais utilizados para avaliação da condição fetal, embora a realização dos testes, mesmo em gestações de risco, não altere desfechos, como a ocorrência de morte perinatal $(46,47)$. Especificamente em relação ao diabetes, estudos que avaliaram o controle metabólico materno a curto e a longo prazo com as medidas do Doppler não revelaram associação entre eles (48). Somente na suspeita de insuficiência placentária modifica-se o algoritmo de avaliação fetal, incluindo a Dopplervelocimetria da gestação (49).

Nos casos de diabetes gestacional com bom controle glicêmico, sem tratamento farmacológico, a avaliação de bem-estar fetal não é necessária até o termo, na ausência de outra indicação. A atual recomendação oficial no Reino Unido (22) é não avaliar rotineiramente o bem-estar fetal nas gestações com diabetes antes das 38 semanas, na ausência de insuficiência placentária. 
O rastreamento para doença hipertensiva da gestação, com a medida da pressão arterial e quando indicado, da relação proteína/creatinina em amostra de urina, é realizado a cada consulta pré-natal (13), pois o risco de pré-eclâmpsia eleva-se em gestações complicadas por diabetes gestacional (5).

O rastreamento de bacteriúria assintomática por urocultura trimestral é recomendado, pois a infecção urinária e a pielonefrite são as causas mais comuns de descompensação metabólica na gestação com diabetes.

\section{MANEJO PERIPARTO}

\section{Risco de parto pré-termo}

O hiperinsulinismo fetal, secundário à hiperglicemia materna, parece estar associado ao retardo da produção do surfactante pulmonar e à hipoxemia fetal crônica, mesmo na ausência de vasculopatia materna. Nos casos de prematuridade em gestações complicadas por diabetes gestacional, portanto, somam-se fatores de risco para disfunção respiratória neonatal grave (50). A necessidade de interrupção da gestação antes das 34 semanas, por indicações fetais ou maternas, indica a administração de corticoide, de forma semelhante ao que ocorre nas gestações com tolerância normal à glicose. As doses preconizadas de corticoide são as mesmas da gestação sem diabetes, mas a idade gestacional limítrofe para sua aplicação poderia, teoricamente, ser ampliada.

A aplicação do corticoide provoca imediata alteração glicêmica, que exige controle metabólico intensivo (7) e frequentemente tratamento suplementar com insulina intravenosa, exigindo internação hospitalar. $\mathrm{O}$ algoritmo apresentado na tabela 4 é empregado para a suplementação de insulina aos tratamentos já em uso pela gestante. A infusão de insulina é realizada por bomba a partir da primeira dose de corticoide e deve ser mantida por, pelo menos, 12 horas após a última dose de corticoide (51).

\section{Momento e via do parto}

Gestantes com controle glicêmico satisfatório e sem complicações obstétricas podem aguardar o início espontâneo do trabalho de parto até 39 semanas (52). Revisão sistemática de estudos sobre o momento do parto em mulheres com diabetes gestacional não mostrou diferença nas taxas de cesariana e de complicações perinatais graves entre o grupo com indução eletiva na $38^{\text {a }}$ semana e o grupo com manejo expectante até a $40^{\mathrm{a}}$ semana, quando avaliados os dois estudos randomiza-
Tabela 4. Suplementação de insulina endovenosa durante corticoterapia antenatal em gestantes com diabetes gestacional em tratamento com insulina

\begin{tabular}{lccccc}
\hline Insulina SC em uso & $\mathbf{<}$ & $\mathbf{4 0} \mathbf{U} / \mathbf{d}$ & $\mathbf{4 1 - 8 0} \mathbf{~ U / d}$ & $\mathbf{8 1 - 1 2 0 ~ U / d ~}$ & $>\mathbf{1 2 0} \mathbf{~} / \mathbf{d}$ \\
\hline \multicolumn{5}{c}{ Dose de insulina suplementar IV (U/hora) } \\
\hline $\begin{array}{l}\text { Glicemia capilar } \\
\text { (mg/dL) }\end{array}$ & Regime A & Regime B & Regime C & Regime D \\
\hline$<108$ & 0 & 0 & 0 & 0 \\
\hline $109-126$ & 0,5 & 1 & 2 & 3 \\
$127-144$ & 1 & 2 & 3 & 5 \\
$145-162$ & 1,5 & 3 & 4 & 7 \\
$163-180$ & 2 & 4 & 6 & 10 \\
$>180$ & 3 & 6 & 8 & 13 \\
\hline
\end{tabular}

Glicemias não satisfatórias por 2 horas consecutivas - regime inicial é trocado pelo subsequente (A para B, B para C, e assim por diante).Glicemia abaixo de $70 \mathrm{mg} / \mathrm{dL}$ - regime é substituído pelo anterior (B para A, C para B, e assim por diante). Modificado de 51.

dos. Entretanto, houve redução da macrossomia e da distocia de ombro no grupo de manejo ativo em relação ao expectante nos quatro estudos observacionais (53). O diagnóstico de diabetes gestacional não indica necessariamente a cesariana, que deve ser reservada aos casos de indicação obstétrica ou de macrossomia fetal suspeita ou comprovada $(8,52)$.

A ultrassonografia para estimativa do peso fetal de nascimento, no final do terceiro trimestre, mostra tendência a superestimar o peso em fetos menores e subestimá-lo em fetos macrossômicos. Na prática, o erro de estimativa excede $14 \%$ do peso real em todas as fórmulas (54), mas o exame pode auxiliar na decisão da via de parto ao identificar fetos com peso acima de 4.250-4.500 g, que apresentam maior risco para distocia de ombro (55).

A interrupção eletiva da gestação antes de completadas as 38 semanas deve ser antecedida por amniocentese para avaliação da maturidade pulmonar fetal com determinação da presença de fosfatidilglicerol, da razão lecitina/esfingomielina $(\geq 3: 1)$ ou da contagem dos corpos lamelares $(\geq 37.000 / \mu \mathrm{L})$ no líquido amniótico (56). Após 38 semanas, não há indicação de procedimento invasivo para avaliação da maturidade fetal.

\section{Manejo no trabalho de parto}

A hiperglicemia materna durante o parto é uma das causas da hipoglicemia neonatal, motivo pelo qual o controle glicêmico deve ser mantido durante todo o parto. Faz-se a monitorização da glicose capilar a cada uma ou duas horas, procurando manter a glicemia entre $70 \mathrm{e}$ $126 \mathrm{mg} / \mathrm{dL}(7,22)$. Valores inferiores a $60 \mathrm{mg} / \mathrm{dL}$ in- 
dicam a necessidade de infusão de solução glicosada a $5 \%(22)$ e, se hiperglicemia, deve ser iniciada a insulinoterapia (7). Não há estudo comparativo entre uso de insulina regular subcutânea intermitente e infusão endovenosa durante o trabalho de parto, e a conduta deve ser individualizada (22). Gestantes com diabetes gestacional em tratamento apenas com dieta não precisam de nenhum cuidado adicional além daqueles aplicados às gestantes normais.

No parto programado, a gestante permanece em jejum e a dose de insulina intermediária matinal pode ser suspensa ou administrada em um terço da dose usual, com monitorização da glicemia capilar. Quando o parto apresenta início espontâneo e a dose de insulina já foi administrada, recomenda-se inicialmente apenas monitorização da glicemia capilar. Em ambas as situações, o emprego de solução glicosada a $5 \%$ ou de insulinoterapia, conforme a avaliação glicêmica, pode ser necessário $(8)$.

\section{Puerpério}

As mulheres com diabetes gestacional provavelmente não terão necessidade de antidiabéticos orais ou insulina após o parto $(2,7,22)$, mas devem ter a glicemia medida antes da alta hospitalar (22).

A amamentação deve ser encorajada $(2,7)$. A ingestão calórica de $1.800 \mathrm{kcal} /$ dia para a nutriz é geralmente suficiente para suprir as necessidades da lactação e permitir a perda gradual de peso (15). Há poucos estudos observacionais sobre o uso de antidiabéticos orais na lactação para as mulheres que necessitarem manter o tratamento. A glibenclamida não foi detectada no leite materno e as glicemias dos bebês foram normais, sugerindo que a droga seja segura e compatível com o aleitamento, nas doses empregadas, apesar do risco teórico de hipoglicemia no recém-nascido (57). A metformina é excretada no leite em quantidade inferior a $1 \%$ da dose materna ajustada pelo peso; apesar disso, não é recomendada na lactação por alguns autores; no entanto, o guia de referência sobre drogas na gestação e lactação (58) registra que a metformina é excretada no leite materno em doses compatíveis com a lactação. A dose da acarbose absorvida sistemicamente é inferior a $2 \%$ da dose administrada, e, mesmo se excretada no leite, a quantidade seria irrelevante. Nateglinida, pioglitazona e rosiglitazona provavelmente tenham pequena excreção no leite materno, mas são consideradas compatíveis com a lactação (58).
Todas as mulheres com diagnóstico de diabetes gestacional devem receber orientação sobre mudanças no estilo de vida, como controle do peso, uso de dieta e prática de exercícios físicos (22). Além disso, devem ser avaliadas no puerpério para a reclassificação da condição metabólica. O teste oral de tolerância com $75 \mathrm{~g}$ de glicose deve ser realizado de seis a oito semanas após o parto $(2,7)$. Menos de $20 \%$ das mulheres com diagnóstico de diabetes gestacional (critério Carpenter-Counstan) realizaram reavaliação da tolerância à glicose no puerpério; dentre estas, $1,4 \%$ persistiu com diabetes (59). No acompanhamento pós-parto do Estudo Brasileiro de Diabetes Gestacional (EBDG), o diagnóstico de diabetes gestacional dobrou o risco de anormalidade na tolerância à glicose 4-8 anos após a gestação-índice (60).

Em conclusão, o diabetes gestacional é condição frequente nas gestações, alcançando 7,2\% em estimativas nacionais (8) e até $17 \%$ se empregado o novo critério diagnóstico sugerido pela IADPSG e referendado pela ADA. O tratamento é recomendado com base nos resultados de ensaios randomizados e as recomendações finais estão sintetizadas na tabela 5 , juntamente com os níveis de evidência.

A maioria das gestantes alcançará os níveis glicêmicos desejados apenas com medidas não farmacológicas; entretanto, cerca de um terço das gestantes necessitará de fármacos no esquema terapêutico por falha no controle glicêmico ou por crescimento fetal excessivo à ultrassonografia.

Diante de vários estudos observacionais e de ensaios clínicos randomizados avaliando não apenas a eficácia da metformina e da glibenclamida, mas também a segurança das medicações e a satisfação das pacientes, torna-se necessário considerar seu uso no diabetes gestacional. Dessa forma, os Serviços de Endocrinologia e de Ginecologia e Obstetrícia do Hospital de Clínicas de Porto Alegre propõem um algoritmo de tratamento conjunto, empregando a metformina como terapia farmacológica inicial. O uso imediato de insulina está indicado nos casos em que exista hiperglicemia acentuada - glicemia de jejum acima de $140 \mathrm{mg} / \mathrm{dL}$ e/ou glicemia em $2 \mathrm{~h}$ acima de $200 \mathrm{mg} / \mathrm{dL}$ - ou, ainda, sintomas de diabetes descompensado, contraindicações à metformina ou preferência da paciente.

Para monitorização do tratamento, utiliza-se a glicemia capilar antes e após as refeições, além da medida da circunferência abdominal fetal após a $28^{\mathrm{a}}$ semana de gestação.

A evolução da gestação até 39 semanas é recomendada, sem necessidade de interrupção precoce, exce- 
Tabela 5. Considerações finais e graus de recomendação

\begin{tabular}{|c|c|}
\hline Considerações & $\begin{array}{c}\text { Grau de } \\
\text { recomendação }\end{array}$ \\
\hline $\begin{array}{l}\text { A terapia nutricional é a primeira opção de tratamento para a } \\
\text { maioria das gestantes }\end{array}$ & A \\
\hline $\begin{array}{l}0 \text { exercício físico deve ser estimulado na ausência de } \\
\text { contraindicações }\end{array}$ & A \\
\hline $\begin{array}{l}\text { A metformina e a glibenclamida são opções terapêuticas com } \\
\text { segurança e eficácia semelhantes à insulina para o controle } \\
\text { glicêmico e desfechos materno-fetais }\end{array}$ & A \\
\hline $\begin{array}{l}\text { A insulinoterapia é o tratamento padrão e é iniciado nas } \\
\text { gestantes que não atingem as metas de controle glicêmico } \\
\text { com dieta e/ou medicação oral ou apresentam fatores } \\
\text { preditores de falha. As insulinas NPH, regular, lispro e } \\
\text { asparte são atualmente recomendadas na gestação }\end{array}$ & A \\
\hline $\begin{array}{l}\text { A monitorização glicêmica é realizada com medida da } \\
\text { glicemia capilar pré- e pós-prandial, com alvos de glicemia } \\
\text { de jejum < } 95 \mathrm{mg} / \mathrm{dL}, 1 \mathrm{~h} \text { pós-prandial < } 140 \mathrm{mg} / \mathrm{dL} \text { e } 2 \mathrm{~h}< \\
120 \mathrm{mg} / \mathrm{dL}\end{array}$ & A \\
\hline $\begin{array}{l}\text { A monitorização do tratamento também deve ser realizada } \\
\text { com a medida ecográfica da circunferência abdominal fetal a } \\
\text { partir da } 28^{\mathrm{a}} \text { semana de gestação, que deve estar abaixo do } \\
\text { percentil } 75\end{array}$ & A \\
\hline $\begin{array}{l}\text { As ultrassonografias de primeiro trimestre para datação e } \\
\text { rastreamento de cromossomopatias (11-14 semanas) e de } \\
\text { segundo trimestre para avaliação morfológica fetal (18-22 } \\
\text { semanas) devem ser realizadas em todas as gestações }\end{array}$ & A \\
\hline $\begin{array}{l}\text { A ecocardiografia fetal pode ser realizada a partir de } 25-26 \\
\text { semanas, porém apresenta custo-efetividade controversa }\end{array}$ & D \\
\hline A monitorização do bem-estar fetal pode ser realizada & D \\
\hline $\begin{array}{l}0 \text { momento e a via de parto devem ter indicação obstétrica. } \\
\text { Diabetes gestacional não é indicação de cesariana }\end{array}$ & B \\
\hline $\begin{array}{l}\text { A g licemia durante o trabalho de parto deve ser mantida entre } \\
70 \text { e } 126 \mathrm{mg} / \mathrm{dL} \text {. Insulinoterapia ou soro glicosado } 5 \% \\
\text { podem ser utilizados }\end{array}$ & B \\
\hline
\end{tabular}

A: Estudos experimentais e observacionais de melhor consistência; B: Estudos experimentais e observacionais de menor consistência; D: Opinião de especialista, diretrizes, estudos em modelos animais.

to nos casos com indicação obstétrica. A indicação da via de parto é obstétrica e as gestantes sem evidência de crescimento fetal excessivo (peso fetal estimado $<$ 4.250-4.500 g) são candidatas ao parto vaginal. $\mathrm{O}$ controle glicêmico periparto é essencial.

Embora a maior parte das mulheres com diabetes gestacional não necessite de antidiabéticos orais ou de insulina após o parto, mudanças no estilo de vida devem ser orientadas visando à prevenção do diabetes tipo 2 . Além disso, é preciso ressaltar a importância da reavaliação do estado metabólico no puerpério, pois apenas uma minoria dessas puérperas a realiza.

Em síntese, o tratamento multidisciplinar estruturado do diabetes gestacional pode proporcionar desfechos maternos e fetais semelhantes aos de populações de baixo risco; a reavaliação e a orientação pós-parto identificam precocemente mulheres com alteração metabólica, possibilitando a adoção de ações de prevenção.

Declaração: os autores declaram não haver conflitos de interesse científico neste estudo.

\section{REFERÊNCIAS}

1. Standards of Medical Care in Diabetes. American Diabetes Association. Position Statement. Diabetes Care. 2011;34(Suppl1):S11-61.

2. International Diabetes Federation. Global Guideline on Pregnancy and Diabetes. 2009. Disponível em: www.idf.org. Acesso em: Dez 2010.

3. Schmidt MI, Matos MC, Reichelt AJ, Forti AC, Lima L, Duncan BB. Prevalence of gestational diabetes mellitus - do the new WHO criteria make a difference? Diabet Med. 2000;17:376-80.

4. IADPSG Consensus Panel. International Association of Diabetes and Pregnancy Study Groups. Recommendations on the Diagnosis and Classification of Hyperglycemia in Pregnancy. Diabetes Care. 2010;33(3):676-82.

5. HAPO Study Cooperative Research Group. Hyperglycemia and adverse pregnancy outcomes. N Engl J Med. 2008;358:1991-2002.

6. Crowther CA, Hiller JE, Moss JR, McPhee AJ, Jeffries WS, Robinson JS; for the Australian Carbohydrate Intolerance Study in Pregnant Women (ACHOIS) Trial Group. Effect of treatment of gestational diabetes mellitus on pregnancy outcomes. N Engl J Med. 2005;352(24):2477-86.

7. Diretrizes da Sociedade Brasileira de Diabetes 2009. $3^{\mathrm{a}}$ Edição. São Paulo. IBSN 978-85-60549-30-6.

8. Reichelt AJ, Oppermann MLR, Schmidt MI. Recomendações da $2^{\mathrm{a}}$ reunião do grupo de trabalho em diabetes e gravidez. Arq Bras End Metabol. 2002;46:574-81.

9. Landon MB, Spong CY,Thom E, Carpenter MW, Ramin SM, Casey $B$, et al. A prospective multicenter randomized treatment trial of mild gestational diabetes. N Engl J Med. 2009;361(14):1339-48.

10. Langer O, Conway DL, Berkus MD, Xenakis EM, Gonzales O. A comparison of glyburide and insulin in women with gestational diabetes mellitus. N Engl J Med. 2000;343(16):1134-8.

11. Rowan JA, Hague WM, Gao W, Battin MR, Moore MP. Metformin versus insulin for the treatment of gestational diabetes. $\mathrm{N}$ Engl $\mathrm{J}$ Med. 2008;358(19):2003-15.

12. Reader DM. Medical nutrition therapy and lifestyle interventions. Diabetes Care. 2007;30(Suppl2):S188-93.

13. Metzger BE, Buchanan TA, Coustan DR, Leiva A, Dunger DB, Hadden $D R$, et al. Summary and recommendations of the fifth international workshop-conference on gestational diabetes mellitus. Diabetes Care. 2007;30(suppl2):S251-60.

14. Artal R, Rosemary B, Catanzaro RB, Gavard JA, Mostello DJ, Friganza JC. A lifestyle intervention of weight-gain restriction: diet and exercise in obese women with gestational diabetes mellitus. Appl Physiol Nutr Metab. 2007;32:596-601.

15. Kaiser L, Allen LH. Position of the American Dietetic Association: nutrition and lifestyle for a healthy pregnancy outcome. J Am Diet Assoc. 2008;108(3):553-61.

16. Major CA, Henry MJ, Veciana M, Morgan MA. The effects of carbohydrate restriction in patients with diet-controlled gestational diabetes. Obstet Gynecol. 1998;91(4):600-4.

17. Moses RG, Barker M, Winter M, Petocz P, Brand-Miller JC. Gestational diabetes: can a low glycemic index diet reduce the need for insulin? A randomized trial. Diabetes Care. 2009;32:996-1000.

18. Peterson CM, Jovanovic-Peterson L. Percentage of carbohydrate and glycemic response to breakfast, lunch, and dinner in women with gestational diabetes. Diabetes. 1991;40(Suppl2):172-4.

19. Knopp RH, Magee MS, Raisys V, BenedettiT. Metabolic effects of hypocaloric diets in management of gestational diabetes. Diabetes. 1991;40(Suppl2):165-71.

20. Rasmussen KM, Yaktine AL; for the Committee to Reexamine IOM Pregnancy Weight Guidelines; Institute of Medicine; National Research Council. Disponível em: www.nap.edu/catalog/12584.html. Acesso em: Dez 2010.

21. Artal R. Exercise: the alternative therapeutic intervention for gestational diabetes. Clin Obstet Gynecol. 2003;46(2):479-87.

22. National Institute for Health and Clinical Excellence. Diabetes in pregnancy. Management of diabetes and its complications from 
preconception to the postnatal period. March 2008. Disponivel em: www.nice.org.uk. Acesso em: Dez 2010.

23. Joint SOGC/CSEP. Clinical Practice Guideline. Exercise in Pregnancy and the Postpartum Period. J Obstet Gynaecol Can. 2003;25(6):516-22.

24. Bonomo M, Cetin I, Pisoni MP, Faden D, Mion E, Taricco E, et al. Flexible treatment of gestational diabetes modulated on ultrasound evaluation of intrauterine growth: a controlled randomized clinical trial. Diabetes Metab. 2004,30:237-43.

25. Kjos SL, Schaffer-Graf U, Sardesi S, Peters RK, Buley A, Xiang $\mathrm{AH}$, et al. A randomized controlled trial using glycemic plus fetal ultrasound parameters versus glycemic parameters to determine insulin therapy in gestational diabetes with fasting hyperglycemia. Diabetes Care. 2001;24:1904-10.

26. Young J, Anwar A. Diabetic medications in pregnancy. Curr Diabetes Rev. 2009;5:252-8.

27. Hemauer SJ, Patrikeeva SL, NanovskayaTN, Hankins GD, Ahmed MS. Role of human placental apical membrane transporters in the efflux of glyburide, rosiglitazone, and metformin. Am J Obstet Gynecol. 2010;202:383.e1-7.

28. Gilbert C, Valois M, Koren G. Pregnancy outcome after first-trimester exposure to metformin: a meta-analysis. Fertil Steril. 2006;86(3):658-63.

29. Nicholson W, Bolen S, Witkop CT, Neale D, Wilson L, Bass E. Benefits and risks of oral diabetes agents compared with insulin women with gestational diabetes. A systematic review. Obstet Gynecol. 2009;113(1):193-205.

30. Bertini AM, Silva JC, Taborda W, Becker F, Lemos Bebber FR, Zucco Viesi JM, et al. Perinatal outcomes and the use of oral hypoglycemic agents. J Perinat Med. 2005;33(6):519-23.

31. Moore TR. Glyburide for the treatment of gestational diabetes. Diabetes Care. 2007;30(Suppl2):S209-13.

32. Rochon M, Rand L, Roth L, Gaddipati S. Glyburide for the management of gestational diabetes: risk factors predictive of failure and associated pregnancy outcomes. Am J Obstet Gynecol. 2006;195(4):1090-4.

33. Nachum Z, Ben-Shlomo I, Weiner E, Shalev E. Twice daily versus four times daily insulin dose regimens for diabetes in pregnancy: randomized controlled trial. BMJ. 1999;319(7219):1223-7.

34. Singh SR, Ahmad F, Lal A, Yu C, Bai Z, Bennett H. Efficacy and safety of insulin analogues for the management of diabetes mellitus: a meta-analysis. CMAJ. 2009;180(4):385-97.

35. Pollex EK, Feig DS, Lubetsky A, Yip PM, Kortn G. Insulin glargine safety in pregnancy. Diabetes Care. 2010;33:29-33.

36. Veciana M, Major CA, Morgan MA, AsratT,Toohey JS, Lien JM, et al. Postprandial versus preprandial blood glucose monitoring in women with gestational diabetes mellitus requiring insulin therapy. N Engl J Med. 1995;333(19):1237-41.

37. Hawkins JS, Casey BM, Lo JY, Moss K, McIntire DD, Leveno KJ. Weekly compared with daily blood glucose monitoring in women with diet-treated gestational diabetes. Obstet Ginecol. 2009;113(6):1307-12.

38. Rowan JA, Gao W, Hague WM, McIntyre HD. Glycemia and its relationship to outcomes in the metformin in gestational diabetes trial. Diabetes Care. 2010;33:9-16.

39. Langer O, Levy J, Brustman L, Anvaegbunam A, Merkatz R, Divon M. Glycemic control in gestational diabetes mellitus--how tight is tight enough: small for gestational age versus large for gestational age? Am J Obstet Gynecol. 1989;161(3):646-53.

40. Gandhi RA, Brown J, Simm A, Page RC, Idris I. HbA1c during pregnancy: its relationship to meal related glycaemia and neonatal birth weight in patients with diabetes. Eur J Obstet Gynecol Reprod Bio. 2008;138:45-8.

41. Nielsen LR, Ekbom $P$, Damm $P$, Glümer $C$, Frandsen MM, Jensen $\mathrm{DM}$, et al. HbA1c levels are significantly lower in early and late pregnancy. Diabetes Care. 2004;27:1200-1.
42. RizzoT, Metzger BE, Burns WJ, Burns K. Correlations between antepartum maternal metabolism and child intelligence. $\mathrm{N}$ Engl $\mathrm{J}$ Med. 1991;325(13):911-6.

43. Schaefer-Graf UM, Wendt L, Sacks DA, Kilavuz O, Gaber B, Metzner $\mathrm{S}$, et al. How many sonograms are needed to reliably predict the absence of fetal overgrowth in GDM pregnancies? Diabetes Care. 2011;34:39-43.

44. Macintosh M, Fleming KM, Bailey JA, Doyle P, Modder J, Acolet $D$, et al. Perinatal mortality and congenital anomalies in babies of women with type 1 or type 2 diabetes in England, Wales, and Northern Ireland: population based study. BMJ. 2006;333(7560):177.

45. Bernard LS, Ramos GA, Fines V, Hull AD. Reducing the cost of detection of congenital heart disease in fetuses of women with pregestational diabetes mellitus. Ultrasound Obstet Gynecol. 2009;33(6):676-82.

46. Grivell RM, Alfirevic Z, Gyte GML, Devane D. Antenatal cardiotocography for fetal assessment. Cochrane Database Syst Rev. 2010,20(1):CD007863.

47. Lalor JG, Fawole B, Alfirevic Z, Devane D. Biophysical profile for fetal assessment in high risk pregnancies. Cochrane Database Syst Rev. 2008;23(1):CD000038.

48. Reece EA, Hagay Z, Assimakopoulos E, Moroder W, Gabrielli S, DeGennaro N, et al. Diabetes mellitus in pregnancy and the assessment of umbilical artery waveforms using pulsed Doppler ultrasonography. J Ultrasound Med. 1994;13(2):73-80.

49. Alfirevic Z, Stampalija T, Gyte GM. Fetal and umbilical Doppler ultrasound in high-risk pregnancies (review). Cochrane Database Syst Rev. 2010;20(1):CD007529.

50. Piper JM, Xenakis EM, Langer O. Delayed appearance of pulmonary maturation markers is associated with poor glucose control in diabetic pregnancies. J Matern Fetal Med. 1998;7(3):148-53.

51. Kaushal K, Gibson JM, Railton A, Hounsome B, New JP, Young RJ. A protocol for improved glycaemic control following corticosteroid therapy in diabetic pregnancies. Diabet Med. 2003;20:73-5.

52. Conway DL. Obstetric management in gestational diabetes. Diabetes Care. 2007;30(Suppl2):S175-9.

53. Witkop CT, Neale D, Wilson LM, Bass EB, Nicholson WK. Active compared with expectant delivery management in women with gestational diabetes: a systematic review. Obstet Gynecol. 2009;113:206-1.

54. Dudley NJ. A systematic review of the ultrasound estimation of fetal weight. Ultrasound Obstet Gynecol. 2005;25(1):80-9.

55. Conway DL, Langer O. Elective delivery of infants with macrosomia in diabetic women: reduced shoulder dystocia versus increased cesarean deliveries. Am J Obstet Gynecol. 1998;178:922-5.

56. DeRoche ME, Ingardia CJ, Guerette PJ, Wu AH, La Sala CA, Mandavilli SR. The use of lamellar body counts to predict fetal lung maturity in pregnancies complicated by diabetes mellitus. Am J Obstet Gynecol. 2002;187:908-12.

57. Feig DS, Briggs GG, Kraemer JM, Ambrose PJ, Moskovitz DN, Nageotte $M$, et al. Transfer of glyburide and glipizide into breast milk. Diabetes Care. 2005;28(8):1851-5.

58. Briggs GG, Freeman RK, Yaffe SJ. Drugs in Pregnancy and Lactation. A reference guide to fetal and neonatal risk. 7th ed. Philadelphia: Lippincott, Williams and Wilkins; 2005.

59. Blatt AJ, Nakamoto JM, Kaufman HW. Gaps in diabetes screening during pregnancy and postpartum. Obstet Gynecol. 2011;117:61-8.

60. Jacob Reichelt AA, Ferraz TM, Rocha Oppermann ML, Costa e Forti A, Duncan BB, Fleck Pessoa E, et al. Detecting glucose intolerance after gestational diabetes: inadequacy of fasting glucose alone and risk associated with gestational diabetes and second trimester waist-hip ratio. Diabetologia. 2002;45:455-7. 